\title{
Malnutrition and venous thromboembolism are specific issues in elderly patients with coronavirus disease 2019
}

\author{
Zheng Qin ${ }^{1 \#}$, Xingjian Wang ${ }^{1 \#}$, Wei Wang ${ }^{1}$ \\ 'Department of Respiratory and Critical Care Medicine, The First Hospital of China Medical University, \\ Shenyang, Liaoning Province, China
}

"Zheng Qin and Xingjian Wang have equally contributed to this manuscript.

Address for Correspondence:

Prof. Wei Wang,

Department of Respiratory and Critical

Care Medicine, The First Hospital of

China Medical University,

155 Nanjing North Street, Heping District,

Shenyang, Liaoning, China, 110001.

E-mail: wwbycmu@126.com

\begin{tabular}{|l|}
\hline Access this article online \\
Website: \\
www.intern-med.com \\
DOI: \\
10.2478/jtim-2021-0027 \\
\hline Quick Response Code: \\
\hline \\
\hline
\end{tabular}

\section{TO THE EDITOR}

Elderly population are more susceptible to coronavirus disease 2019 (COVID-19) and more likely to develop into severe cases after infection. Attention is usually paid to co-infection and multiple comorbidities in their clinical courses, but other important issues may sometimes be ignored. We specially address here the risk of venous thromboembolism and malnutrition in elderly COVID-19 patients according to our experience in the treatment of an 82-yearold female case.

The patient was referred to Wuhan General Hospital because of fever $\left(38.8^{\circ} \mathrm{C}\right)$, dry cough, and chest distress on February 7, 2020 (day 1). Chest computerized tomography scan showed bilateral subsegmental opacities, patchy consolidation, and enlarged mediastinal lymph nodes. Arbidol hydrochloride (0.2 g, three times a day), moxifloxacin (0.4 g, once a day), and sulperazone (cefoperazone $1.0 \mathrm{~g}$ and sulbactam $0.5 \mathrm{~g}$, twice a day) were administrated, and noninvasive ventilator was applied. Because of her poor nutritional status (NRS2002 score $=5$ ), intact protein enteral nutrition powder was added to her regimen. Her fever persisted from day 2 to day 3 with the highest temperature of $39.0^{\circ} \mathrm{C}$, and the level of albumin decreased continuously $(24.2 \mathrm{~g} / \mathrm{L}$ on day 3). A supplement of albumin was given intravenously on day 4 (35 g) and day 7 (20 g). Her temperature has been controlled under $37^{\circ} \mathrm{C}$ since day
10 , and chest radiology on day 15 indicated the alleviation of pulmonary infiltration. Negative results were shown in the viral nucleic acid detections on day 14 and day 16 , and she was discharged on day 17. Notably, during her hospitalization, D-dimer level increased significantly to $6.35 \mu \mathrm{g} / \mathrm{mL}$ on day 6 . She did not present the symptom of dyspnea. Pulmonary embolism was excluded by computed tomography pulmonary angiography (CTPA), but ultrasonographic examination showed thromboembolism in bilateral gastrocnemius veins. Lowmolecular-weight heparin (4000 IU, twice a day) was subsequently put on her regimen, and her D-dimer level reduced to $1.01 \mu \mathrm{g} /$ $\mathrm{mL}$ on day 16. Rivaroxaban was suggested to take for 3 months after discharge with the routine examination of coagulation indicators.

Nutritional status is easily undermined after the infection of SARS-CoV-2 due to the impaired appetite, the consumption of protein in immune response, and the impact of virus on gastrointestinal tract. ${ }^{[1]}$ Elderly patients with COVID-19 are more susceptible to malnutrition because of age-related muscle loss, chewing problem, and psychosocial disadvantages, ${ }^{[2]}$ which compromises immunocompetence and leads to their longer hospital stay as well as increased disease severity. ${ }^{[3]}$ On the other hand, hypoxia, inflammatory, and procoagulant state, as well as venous stasis caused by immobility, contribute to the development of venous thromboembolism (VTE) in COVID-19. ${ }^{[4]}$ Besides, advanced 
age is an intrinsic risk factor for VTE, ${ }^{[5]}$ which suggests an even higher incidence of thrombosis complications in elderly patients. However, many VTE cases in COVID-19 are asymptomatic, ${ }^{[5]}$ and the complex clinical courses of elderly patients may further lead to the underestimation of this condition; therefore, monitoring VTE in their treatment with D-dimer, ultrasonography, and CTPA is indispensable. In conclusion, malnutrition and VTE are prevalent but easily underestimated in elderly patients with COVID-19. Special attention should be paid to these issues in clinical practice, and further studies are required to determine the precise screening and tailored intervention approaches for malnutrition and VTE, especially in elderly patients.

\section{Conflict of Interest}

Wei Wang is an Associate Editor-in-Chief of the journal. This article was subject to the journal's standard procedures, with peer review handled independently of this editor and his research groups.

\section{Source of Funding}

This work was supported by the emergency project of Liaoning Provincial Science and Technology Department, No. 2020JH2/10300003.

\section{REFERENCES}

1. Kopel J, Perisetti A, Gajendran M, Boregowda U, Goyal H. Clinical Insights into the Gastrointestinal Manifestations of COVID-19. Dig Dis Sci 2020; 65: 1932-9.

2. Liu A, Cong J, Wang Q, Mei Y, Peng Y, Zhou M, et al. Risk of Malnutrition Is Common in Patients with Coronavirus Disease 2019 (COVID-19) in Wuhan, China: A Cross-sectional Study. J Nutr 2021;151:1591-6.

3. Liu G, Zhang S, Mao Z, Wang W, Hu H. Clinical significance of nutritional risk screening for older adult patients with COVID-19. Eur J Clin Nutr 2020;74:876-83.

4. García-Ortega A, de la Rosa D, Oscullo G, Castillo-Villegas D, López-Reyes R, Martínez-García MÁ. Coagulation disorders and thromboembolic disease in COVID-19: review of current evidence in search of a better approach. J Thorac Dis 2021; 13:1239-55.

5. Cai C, Guo Y, You Y, Hu K, Cai F, Xie M, et al. Deep Venous Thrombosis in COVID-19 Patients: A Cohort Analysis. Clin Appl Thromb Hemost 2020; 26: 1076029620982669 .

How to cite this article: Qin Z, Wang X, Wang W. Malnutrition and venous thromboembolism are specific issues in elderly patients with coronavirus disease 2019. J TransI Intern Med 2021; 9: 223-4. 